\title{
Recientes Políticas de Inmigración e Integración en los Países Nórdicos. Dinámicas transnacionales y discursos nacionales ante la inmigración forzada y económica
}

\author{
Ángel Manuel Ahedo Santiesteban*
}

\section{Resumen}

Los países nórdicos han tenido una fuerte tradición internacionalista y humanitaria en sus políticas de refugio. En las últimas décadas sus políticas de inmigración e integración han experimentado: en primer lugar una mayor interrelación, por ejemplo, las políticas de integración son un elemento principal para construir la imagen del país respecto a potenciales inmigrantes o refugiados; y en segundo lugar un creciente carácter intervencionista, restrictivo y excluyente, más claramente desde la crisis de refugiados en 2015 . Estas recientes políticas de inmigración-integración se llevaron a cabo a través de unas dinámicas en dos niveles: un primero de debates y políticas transnacionales, principalmente en la Unión Europea; y un segundo sobre discursos nacionales sobre la inmigración y modelos nacionales de ideología y ciudadanía que definen el marco de expectativa de la integración de los inmigrantes. El artículo analiza algunos de los factores relevantes que explican estos discursos y políticas, como la influencia de los partidos antiinmigración, el papel de los nuevos medios digitales y el efecto discursivo de la comunidad investigadora y experta.

\section{Palabras clave}

Crisis de refugiados; políticas de inmigración; políticas de integración; extranjería; ciudadanía.

\section{实-}

\section{TitLE}

Recent Immigration and Integration Policies in the Nordic Countries. Transnational dynamics and national discourses faced with forced and economic immigration

\begin{abstract}
The Nordic countries, characterized by developed welfare states, had a strong tradition of a humanitarian and an internationalist refugee policy. In the last decades, however, the immigration and integration policies have become: firstly increasingly interrelated, for example, integration policies are a basic element to construct country's external image, with regards to potential immigrants and refugees; and secondly more interventionist, restrictive and exclusive, especially since the so-called refugee crisis of 2015. These immigrationintegration policies take place within dynamics at two levels: a first level of transnational debates and policy processes, and a second level of national discourses on immigration and national ideologies and models of citizenship that define the frame of expectation for the integration of immigrants. This article looks at some of the relevant factors that have been the main drivers of these discourses and policies, such as the influence of anti-immigrant parties, the growing role of digital media, and the research-academic community's discursive effects.
\end{abstract}

\section{KeyWords}

Refugees' crisis; immigration policies; integration policies; foreigners; citizenship.

\section{*Ángel Manuel AHEDO SANTIESTEBAN, Profesor a tiempo parcial del Departa- mento de Sociología y Ciencia Política en la Facultad de Ciencias Sociales de la Universidad de Copenhague (Dina- marca)-}

\section{Agradecimientos:} Gracias a las personas que han evaluado este artículo por sus críticas, comentarios y sugerencias.

\section{Recibido:}

2017-04-21

Aceptado:

2017-09-25

DOI: $10.15366 /$ relacionesinternacio nales2017.36.001 


\section{ntroducción}

La migración es un fenómeno histórico y de creciente relevancia global. No hay una teoría definitiva sobre la migración contemporánea. King ${ }^{1}$ sugiere que las diferentes teorías económicas, sociales y demográficas deberían relacionar la migración con los procesos sociales, económicos y políticos globales, y con la noción de acceso a la movilidad, así como integrar el análisis de la migración con la post-migración o integración.

Los países europeos occidentales se han convertido en sociedades de inmigración. Messina propone tres oleadas y tipos de inmigración: ${ }^{2}$ una primera oleada de inmigración laboral (1945-1979): trabajadores, mayormente hombres, fueron invitados a contribuir al crecimiento industrial; una segunda de inmigración secundaria y establecimiento permanente (1973-2007): se procedió a la reunificación familiar de los trabajadores invitados y a su residencia permanente; y una tercera de inmigración ilegal y forzada (1989-2007): por los desequilibrios del capitalismo y los conflictos en países asiáticos y africanos. Desde el 2007 el segundo y tercer tipo han aumentado, con un creciente número de refugiados. Para Castles $^{3}$ la migración forzada, de refugiados, buscadores de asilo, desplazamientos internos y los provocados por proyectos de desarrollo, era ya en los años 1990 una relevante realidad en las relaciones Norte-Sur y la economía política global.

Este artículo analiza de forma integrada la inmigración económica (más o menos voluntaria) y la inmigración forzada (principalmente refugiados), y opta por una complementariedad entre los estudios de migración y los estudios de refugiados o de migración forzada. ${ }^{4}$ Hay dos motivos principales: el primero que en la Europa noroccidental, y más en los países nórdicos, ${ }^{5}$ con una tradición de política de refugio, la migración económica está altamente relacionada con la inmigración forzada; el segundo que en los debates públicos-políticos sobre la inmigración en estos países no se suele diferenciar entre inmigrantes económicos y refugiados, y de forma interesada y estratégica, los dos fenómenos son incluidos dentro del tema general de inmigrantes o extranjeros, con atención en los no-occidentales.

Desde los años setenta y ochenta muchos países europeos comenzaron a desarrollar políticas de integración, desde un pasado de prácticas de integración asimilacionistas y de regulaciones liberales en lo que respecta a los derechos civiles y políticos. A finales de los noventa las políticas de integración iniciaron el llamado "giro cívico", con nuevas y exigentes condiciones para optar a los derechos de ciudadanía y nacionalidad. ${ }^{6}$ Estas políticas han sido también adoptadas por los gobiernos de los países nórdicos, que presentan estas políticas como realistas con el objetivo de mejorar la integración de los inmigrantes, reducir y contro-

1 KING, Russell, Theories and typologies of migration: An overview and a primer. Malmö University, Malmö Institute for Studies of Immigration, Diversity and Welfare (MIM), Malmö 2012.

2 MESSINA, Anthony M., The logics and politics of post-WWII migration to Western Europe, Cambridge University Press, Cambridge, 2007.

3 CASTLES, Stephen, "Towards a sociology of forced migration and social transformation" en Sociology, vol. 37, no 1,2003 , pp. 13-34.

4 FIDDIAN-QASMIYEH, Elena, LOESCHER, Gil, LONG, Katy y SIGONA, Nando (ed.), The Oxford handbook of refugee and forced migration studies, Oxford University Pres, Oxford, 2014.

5 Escandinavo se refiere a Dinamarca, Noruega y Suecia; y nórdico incluye Finlandia.

6 JOPPKE, Christian, "Beyond national models: Civic integration policies for immigrants in Western Europe" en West European Politics vol. 30, no 1, 2007, pp. 1-22. 
lar la llegada de personas extranjeras, y preservar sus estados de bienestar. Su cercanía y similitud favorecen la difusión y la influencia mutua. Cada país nórdico ha tenido su proceso de debate y discursos, dentro de su modelo ideológico de integración y ciudadanía.

La inmigración en los países nórdicos ha tendido unos rasgos diferenciados. No han sido poderes imperiales modernos. En los años cincuenta y sesenta realizaron programas de "trabajadores-inmigrantes invitados". Desde los años setenta, junto a una creciente ayuda al desarrollo, llevaron a cabo humanitarias políticas de refugio. Los países nórdicos han sido atractivos por su sistema público de solidaridad y su generosa política de refugiados. Sus estados de bienestar fueron construidos desde una base de homogeneidad étnico-cultural, pero con la inmigración ha aumentado la diversidad étnico-cultural. Desde finales de los años ochenta y noventa ha habido un progresivo endurecimiento de las políticas de inmigración-integración.

El artículo analiza y compara el surgimiento de estas nuevas políticas de inmigración e integración en los países nórdicos. Para su comprensión se analizan dos factores: en primer lugar los discursos dominantes en el debate público-político, con atención a tres tipos de actores: los partidos políticos y específicamente los partidos antiinmigración; los medios de comunicación y la emergencia de los medios sociales y digitales; y el papel de la comunidad investigadora o experta; y en segundo lugar los modelos o tipos nacionales de integración, ya que cada estado-nación moderno ha desarrollado una forma particular de entender la plena ciudadanía. En la segunda sección se presentan los marcos teóricos y conceptuales. En la tercera sección se muestran algunos datos evolutivos sobre el contexto inmigratorio y político de estos países. En la cuarta sección se presenta una nota metodológica. En las sucesivas secciones se analiza cada país de forma individual, para finalizar con las conclusiones.

\section{Las políticas de inmigración e integración en los estados de bienestar nórdicos}

Aunque la inmigración y la integración de los inmigrantes se pueden analizar de forma diferenciada, a nivel de discurso y de debate púbico-político en los países europeos occidentales la cuestión de la inmigración está crecientemente ligada a la integración, y las dos políticas están cada vez más interrelacionadas. ${ }^{7}$ Por ejemplo, en el discurso crítico con la inmigración se suele pensar que una forma de reducir el número de inmigrantes y de solicitudes de asilo es endureciendo las condiciones de vida y de integración de los mismos.

\subsection{Políticas de inmigración e integración}

La política de inmigración tiene dos objetivos: controlar las entradas en el territorio nacional (o en el espacio Schengen, a nivel supranacional), y regular las autorizaciones por motivos de residencia, estudios o trabajo, reagrupación familiar o refugio. Respecto a la situación de refugiado y de solicitantes de asilo, de las tres soluciones convencionales (integración local, repatriación y reubicación), en Europa occidental la integración ha sido claramente la más relevante; la repatriación tras la denegación de la solicitud ha aumentado en algunos países después de la fuerte llegada de solicitantes de asilo en 2014-2015.

La política de integración regula el modo en que la persona extranjera se convierte

7 GEDDES, Andrew y SCHOLTEN, Peter, The politics of migration and immigration in Europe, Sage, London, 2016. 
en un ciudadano con plenos derechos. Pero, ¿cuándo y cómo se considera a una persona extranjera integrada en la sociedad de acogida? La integración tiene varios niveles y dimensiones: en primer lugar políticamente, desde el respeto de la legalidad hasta participar en asociaciones civiles; en segundo lugar económicamente, desde la participación en cursos ocupacionales hasta la participación laboral activa; y en tercer lugar culturalmente, desde aprender y usar el idioma hasta la participación en las tradiciones y prácticas culturales del país. La integración mínima correspondería a un modelo de multiculturalismo, mientras que la integración máxima sería una asimilación. Entre estas dos caben situaciones de republicanismo o acomodación, donde la mayoría de la sociedad acepta las diferencias sustantivas del individuo y su grupo cultural dentro de las reglas comunes de ciudadanía política. Más allá de estos tres tipos, cada sociedad o estado-nación tiene su propia expectativa de lo que es ser una persona integrada con derecho a la plena ciudadanía.

\subsection{Dinámicas y discursos transnacionales}

Desde finales de los años noventa muchos países europeos han ido adoptando políticas de integración con un mayor control, intervención y restricción. Estas políticas han sido conceptualizadas como un "giro cívico". La primera ley fue en los Países Bajos en 1998 (ley de integración del recién llegado), y luego fueron adoptadas en Alemania, Francia, y Bélgica, para posteriormente extenderse a otros países europeos. El objetivo explícito de estas políticas es convertir a las personas extranjeras en ciudadanos integrados de manera rápida y efectiva. A los iniciales programas de introducción han seguido medidas orientadas a la participación activa en el mercado laboral, programas educativos (cursos y exámenes) obligatorios, cuya superación cualifica para acceder a derechos sociales y civiles como la nacionalidad, la naturalización e incluso para la reagrupación familiar; también se han tomado medidas restrictivas respecto a la reagrupación familiar. La Unión Europea (UE) también ha acompañado este giro, promoviendo por ejemplo el derecho a trabajar de los refugiados y los solicitantes de asilo en la nueva revisada Directiva de Recepción del 2013, mientras que en general su política de refugio destaca por su poca coordinación y armonización, y por respetar la autonomía de los estados miembros a la hora de hacer leyes más restrictivas.

Para autores como Joppke, este giro "cívico" responde a dos dinámicas transnacionales: a la expansión y desarrollo de la ideología neoliberal con fuerte peso en lo económico, que acoge a su vez ideas neonacionalistas sobre la ciudadanía y la relación entre individuo/ inmigrante y el estado; y al surgimiento, crecimiento e influencia de los partidos políticos antiinmigración, mayormente populistas y conservadores. El giro cívico es así una tendencia transnacional convergente, desde una confluencia en la interpretación del fenómeno, y la difusión de políticas públicas, siendo más claro en el caso de la Unión Europea.

Para Goodman", estas nuevas políticas "cívicas" de integración no han sido tan convergentes, y destaca tres diferencias. Unos países son más activos y precoces que otros en la adopción de estas políticas. Cada país adopta las políticas desde su modelo de integración y su concepción de ciudadanía, es decir, desde sus tradiciones institucionales, y el cambio de esas instituciones requiere amplios acuerdos y consensos. La influencia de los partidos antiin-

8 GOODMAN, Sara Wallace, "Fortifying citizenship: policy strategies for civic integration in Western Europe." en World Politics vol. 64, no 04, 2012, pp. 659-698. 
migración es desigual, y es más conveniente considerar el sistema o constelación de partidos o el discurso político dominante en el país.

Los estudios comparados han analizado también los efectos de estas políticas. En base a datos cuantitativos, Goodman y Wright ${ }^{9}$ concluyen que estas políticas "cívicas" no conllevan una mejor integración, y parecen dificultar la propia integración y desincentivar la inmigración y el refugio. Según Simonsen, ${ }^{10}$ estas políticas tienen el efecto contrario de generar un menor sentimiento de pertenencia a la nación de acogida, incluso entre los que han cumplido todos los requisitos y tienen la ciudadanía o la residencia permanente. El mayor efecto de estas políticas parece ser simbólico: internamente, para indicar a la ciudadanía que el gobierno tiene control de la situación y herramientas de intervención; y externamente, para desincentivar la emigración al país.

\subsection{Discursos y modelos nacionales}

Las políticas de inmigración-integración son el resultado de procesos de debate públicos empíricos y normativos en torno a la problematización política del fenómeno, es decir, sobre lo que es y debe ser la inmigración y la integración. Los discursos dominantes se refieren a las ideas, creencias y argumentos que dominan la interpretación normativa y política de la cuestión. En la teoría crítica del discurso político Van Dijk propone una visión de la ideología en términos de cognición social, a modo de estructuras básicas que organizan las representaciones sociales y las categorías básicas de un grupo social, como los criterios de pertenencia, actividades, objetivos, normas, valores, y relaciones a otros grupos y recursos; estas ideologías y sus representaciones sociales se convierten en modelos mentales de situaciones y eventos concretos, que son la base del discurso. ${ }^{11}$ Van Dijk, en sus estudios sobre los discursos racistas, ha enfatizado el papel de las élites y los actores políticos e institucionales. ${ }^{12}$ En base a estas conceptualizaciones, los dos factores clave a analizar son los actores principales de los discursos dominantes y los modelos nacionales de integración.

Para analizar los discursos dominantes nacionales sobre inmigración-integración se destacan tres tipos de actores. Primero, el sistema de partidos políticos, los cuales lideran el debate político, y en concreto la influencia de los grupos y partidos políticos antiinmigración. Segundo, los medios de comunicación, con especial atención a los medios más populares con altas audiencias y los emergentes medios sociales y digitales. Tercero, la contribución de los académicos e investigadores, los cuales tienen dos funciones: primero como insumo de conocimiento sobre el fenómeno, a modo de referencia para las políticas públicas; según Scholten et al., ${ }^{13}$ los gobiernos y partidos políticos tienden a usar los análisis y argumentos de los

9 GOODMAN, Sara Wallace y WRIGHT, Matthew, "Does mandatory integration matter? Effects of civic requirements on immigrant socio-economic and political outcomes." En Journal of Ethnic and Migration Studies vol. 41, no 12, 2015, pp. 1885-1908.

${ }^{10}$ SIMONSEN, Kristina B, "Does citizenship always further immigrants' feeling of belonging to the host nation? A study of policies and public attitudes in 14 Western democracies" en Comparative Migration Studies vol.5.1, $\mathrm{n}^{\circ}$ 3, 2016

${ }^{11}$ VAN DIJK, Teun, "Ideologies, racism, discourse: Debates on immigration and ethnic issues" en WAL, Jessika ter y VERKUYTEN, Maykel (ed.), Comparative perspectives on racism, Ashgate, 2000, pp. 91-116 (113)

12 VAN DIJK, Teun, "Discurso de las élites y racismo institucional" en LARIO, Manuel (ed.), Medios de comunicación e inmigración, CAM-Obra Social, Murcia, 2006, pp. 15-34.

13 SCHOLTEN, Peter, ENTZINGER, Han y PENNINX, Rinus, "Research-policy dialogues on migrant integration in Europe: comparison and conclusions", en SCHOLTEN, Peter y ENTZINGER, Han (ed.), Integrating Immigrants in 
investigadores y expertos para legitimar y sustanciar sus posturas y propuestas; y segundo como autoridad pública que ofrece guía y orientación a la sociedad. En el tema de la inmigración e integración la imparcialidad científica es difícil dada la presión social y los diversos sesgos. En este sentido los análisis de realidades nacionales por investigadores no-nacionales pueden ser útiles.

El modelo o tipo nacional de integración se refiere a la ideología canónica de lo que supone ser un ciudadano con plenos derechos. Cada estado-nación tiene su particular ideal de lo que es ser un ciudadano integrado, en un continuum que recorre características económicas, políticas, sociales y culturales. Los estados-nación actuales se dirimen entre las tendencias liberales que priorizan criterios económicos y políticos, y las tendencias nacionalistas que priorizan lo cultural y étnico. Según los criterios para acceder a la ciudadanía se pueden identificar tres formas de integración: social, centrada en la participación socio-económica y laboral; cultural, centrada en el idioma y el conocimiento histórico y cultural del país; y política, el tradicional criterio liberal de acceder a la ciudadanía dentro de la cultura democrática moderna.

\subsection{Estados de bienestar nórdicos y debate público-político sobre inmigración}

La inmigración e integración en los países nórdicos ha tendido unos rasgos diferenciados. No han sido poderes imperiales modernos. En los años cincuenta y sesenta realizaron programas de "trabajadores-inmigrantes invitados". Desde los años setenta, junto a una creciente ayuda al desarrollo, llevaron a cabo humanitarias políticas de refugio. Los países nórdicos han sido atractivos por su sistema público de solidaridad y su generosa política de refugiados. Sus estados de bienestar fueron construidos desde una base de homogeneidad étnico-cultural, y con la inmigración aumenta la diversidad étnico-cultural. La inmigración ha sido vista de forma creciente como un problema para este modelo de estado de bienestar. ${ }^{14} \mathrm{El}$ argumento de la inmigración como amenaza del bienestar de la sociedad de acogida es quizá uno de los argumentos (o mitos) más generalizados.

Desde finales de los años ochenta y noventa ha habido un progresivo endurecimiento de las políticas de inmigración-integración. En los años ochenta y noventa, algunas autoridades, políticos y un sector de la opinión pública comenzaron a indicar su preocupación por el efecto de los flujos de inmigración sobre el sistema de bienestar. Junto a las diferencias religiosas y culturales, la alta concentración residencial de los inmigrantes y refugiados, y los problemas en el sistema educativo-escolar, uno de los focos principales del debate se centró en la baja participación de los inmigrantes-refugiados en el mercado de trabajo. Se argumentaba que el estado de bienestar era quizá un desincentivo a la participación laboral de estos grupos, y así se justificó una mayor intervención de los gobiernos para promover su actividad laboral.

Sin embargo, las autoridades, los políticos y la sociedad, salvo en Suecia, han tenido problemas para reconocer sus propias prácticas exclusivas en el mercado laboral, y por tanto

Europe, Springer International Publishing, 2015, pp. 315-336.

${ }^{14}$ BROCHMANN, Grete y HAGELUND, Anniken, "Migrants in the Scandinavian welfare state. The emergence of a social policy problem" en Nordic Journal of Migration Research, vol. 1, no 1, 2011, pp. 13-24. 
su parte de responsabilidad en la baja participación laboral de estas personas. Este es un ejemplo de la importancia del debate público-político sobre la interpretación de la realidad. El creciente carácter restrictivo de las políticas de inmigración e integración debe entenderse en última instancia por un aumento del apoyo a estas ideas en la opinión pública. Para un mejor análisis es necesario contextualizar los actores seleccionados en los países nórdicos.

El sistema de partidos políticos de los países nórdicos tiene tres características comunes: en primer lugar el desarrollo de partidos antiinmigración y populistas, con tendencia a colaborar con los partidos de centro-derecha, y a liderar el neonacionalismo; ${ }^{15}$ en segundo lugar la existencia de fuertes partidos socialdemócratas que impulsaron políticas humanitarias en materia de refugio y asilo, y que han ido modificando sus posturas; y en tercer lugar la importancia de la administración pública municipal, aunque en las recientes políticas de integración se ha producido una centralización. ${ }^{16}$

Sobre el sistema de medios de comunicación, estos países tienen una tradición de sistema pluralista de medios de comunicación. Los medios privados con diferentes ideologías y tendencias (de ideología liberal-conservadora y de ideología progresista) compiten con medios públicos de calidad con profesionales altamente autónomos. Cabe destacar el auge de los medios digitales, en estas sociedades avanzadas en el uso de las nuevas TIC e internet. La relación entre los medios de comunicación más o menos populistas y el neonacionalismo antiinmigración de extrema-derecha ha sido una tendencia común a la Europa occidental ${ }^{17}$.

Finalmente, en estos países los investigadores, expertos y académicos tienen dos principales roles: como investigadores independientes ofrecen a la sociedad su guía y orientación para entender y afrontar los problemas; y como asesores expertos al estado o gobierno de cara a políticas públicas, la cual es parte de su tradicional contribución a la cultura nórdica de consenso institucional. Según Jørgensen, ${ }^{18}$ en Suecia los científicos sociales han sido muy influyentes en la agenda de las políticas de integración, y en Dinamarca el conocimiento científico ha sido utilizado selectivamente para legitimar posturas políticas.

\section{El nuevo contexto nórdico: inmigración y partidos antiinmigración}

Se ofrece una evolución cuantitativa de la inmigración y de los partidos políticos antiinmigración. No se incluyen las prácticas sociales relativas a la integración, como la segregación espacial o residencial, las prácticas laborales que afectan a la participación laboral de los inmigrantes, o las diferencias escolares-educativas. Estas cuestiones, recurrentes en los debates, requieren otro tipo de análisis.

${ }^{15}$ BERGMANN, Eirikur, Nordic Nationalism and Right-Wing Populist Politics, Palgrave Macmillan UK, Basingstoke, 2017; HELSSTRÖM, Anders, Trust us: Reproducing the nation and the Scandinavian nationalist populist parties, Berghahn Books, Nueva York, 2016.

${ }^{16}$ EMILSON, Henrik, "A national turn of local integration policy: multi-level governance dynamics in Denmark and Sweden" en Comparative Migration Studies, vol. 3.1, no 7, 2015.

${ }^{17}$ ELLINAS, Antonis, The Media and the Far Right in Western Europe: Playing the Nationalist Card, Cambridge University Press, Cambridge, 2010.

18 JØRGENSEN, Martin Bak, "Understanding the research-policy nexus in Denmark and Sweden: The field of migration and integration" en The British Journal of Politics and International Relations, vol. 13, no 1, 2011, pp. 93-109. 


\subsection{Evolución cuantitativa de la inmigración}

Hasta comienzos de los noventa la inmigración era principalmente de trabajadores invitados y refugiados. Desde entonces la inmigración económica y forzada continuó, y desde 2005 el crecimiento fue mayor en Suecia y Noruega que en Dinamarca y Finlandia (Tabla 1).

Tabla 1. Porcentaje de personas residentes nacidas en el extranjero 1990-2015

\begin{tabular}{|l|c|c|c|c|c|c|}
\hline & $\mathbf{1 9 9 0}$ & $\mathbf{1 9 9 5}$ & $\mathbf{2 0 0 0}$ & $\mathbf{2 0 0 5}$ & $\mathbf{2 0 1 0}$ & $\mathbf{2 0 1 5}$ \\
\hline Suecia & 7,5 & 9 & 10 & 11 & 15 & 17 \\
\hline Dinamarca & 4 & 5 & 6 & 7,5 & 9 & 11 \\
\hline Noruega & 5 & 6 & 7,5 & 9 & 12,5 & 15 \\
\hline Finlandia & 1 & 1,5 & 2 & 4 & 5 & 6,5 \\
\hline
\end{tabular}

Fuente: Informe Indvandring i Danmark (Inmigración en Dinamarca) Danmarks Statistik, 2016

En la estadística oficial de estos países se han desarrollado nuevas categorías políticamente controvertidas como: descendientes de inmigrantes (personas nacidas en el país, pero ninguno de los progenitores ha nacido en el país ni tiene la nacionalidad del país); y el origen occidental o no-occidental de la población extranjera: los países occidentales son la UE, Noruega, Suiza, Canadá, EEUU, Australia y Nueva Zelanda. En la Tabla 2, la categoría de no-occidental en Suecia era el $11 \%$, en Dinamarca el $6 \%$, en Noruega el 7,5\% y en Finlandia un porcentaje muy bajo. Estas categorías y datos han generado nuevos argumentos en los discursos.

Tabla 2. Porcentaje de personas nacidas en el extranjero 2016, según origen.

\begin{tabular}{|l|c|c|c|c|}
\hline & Suecia & Dinamarca & Noruega & Finlandia \\
\hline Total & 17 & 11 & 15 & 6,5 \\
\hline No-occidental & 10,8 & 6,3 & 7,5 & 1,50 \\
\hline Occidental & 6,2 & 4,7 & 7,5 & 5,75 \\
\hline
\end{tabular}

Fuente: Informe Indvandring i Danmark (Inmigración en Escandinavia), Danmarks Statistik

En la Tabla 3 se observa respecto a los países nórdicos: la tradición de recepción de refugiados, los altas cifras en el 2014 (en Suecia y Dinamarca), 2015 (medido en relación a la población, Suecia fue el primer país, Noruega, cuarto, y Finlandia, quinto), y la marcada reducción en 2016, que en el 2017 ha descendido aún más. 
Tabla 3. Número absoluto de solicitantes de asilo en la Unión Europea (selección de países)

\begin{tabular}{|l|r|r|r|r|r|r|r|}
\hline & $\mathbf{2 0 1 0}$ & $\mathbf{2 0 1 1}$ & $\mathbf{2 0 1 2}$ & $\mathbf{2 0 1 3}$ & $\mathbf{2 0 1 4}$ & $\mathbf{2 0 1 5}$ & $\mathbf{2 0 1 6}$ \\
\hline UE 28 & 259400 & 309040 & 335290 & 431090 & 626960 & 1322825 & 1258865 \\
\hline Alemania & 48475 & 53235 & 77485 & 126705 & 202645 & 476510 & 745155 \\
\hline Francia & 52725 & 57330 & 61440 & 66265 & 64310 & 76165 & 83485 \\
\hline R. Unido & 24335 & 26915 & 28800 & 30585 & 32785 & 40160 & 38785 \\
\hline Italia & 10000 & 40315 & 17335 & 26620 & 64625 & 83540 & 122960 \\
\hline España & 2740 & 3420 & 2565 & 4485 & 5615 & 14780 & 15755 \\
\hline Suecia & 31850 & 29650 & 43855 & 54270 & 81180 & 162450 & 28790 \\
\hline Dinamarca & 5065 & 3945 & 6045 & 7170 & 14680 & 20935 & 6180 \\
\hline Noruega & 10015 & 8990 & 9675 & 11930 & 11415 & 31110 & 3485 \\
\hline Finlandia & 3085 & 2915 & 3095 & 3210 & 3620 & 32345 & 5605 \\
\hline
\end{tabular}

Fuente: Eurostat (Unión Europea)

Estos datos dibujan el contexto mínimo de realidad e información sobre el que se problematiza el tema, y se construyen los discursos y los argumentos políticos.

\subsection{Evolución política y partidos antiinmigración}

En las dos últimas décadas ha habido alternancia de gobiernos de centro-izquierda y centro-derecha-liberales. Han surgido partidos políticos antiinmigración que desde el 2010 en las elecciones parlamentarias han recibido más del $13-15 \%$ de los votos (Tabla 4).

En Suecia en los años ochenta el partido Nueva Democracia desarrolló a nivel local un discurso antiinmigración y nacionalista. Cuando este partido entró en crisis se creó el SD (Demócratas Suecos) en 1988. Aunque criticado por su relación con el histórico movimiento fascista sueco, el SD ha crecido en apoyo electoral. En 2014 los resultados electorales crearon una situación compleja. Ni el bloque de centro-izquierda ni el de centro-derecha tenía mayoría en el parlamento, y el SD podía decidir la balanza. Sin embargo, los partidos de los dos bloques acordaron no colaborar con el SD, el cual quedó marginado en el parlamento.

En Dinamarca, el partido del pueblo danés DF, creado en 1995 como una derivación del antiguo partido del progreso, liberal, antiimpuestos y populista, ha ido creciendo en apoyo electoral; dio apoyo parlamentario a los gobiernos de centro-derecha liberal entre 2001 y 2011, y desde 2015. En 2015 alcanzó su mayor nivel de apoyo con un $21 \%$ de los votos, convirtiéndose en el segundo partido más votado después del partido socialdemócrata. 
Tabla 4. Evolución de los partidos anti-inmigración (\% de votos en elecciones generales)

\begin{tabular}{|l|l|l|l|l|}
\hline & Suecia & Dinamarca & Noruega & Finlandia \\
\hline Hasta 1990 & 1988: SD & & $\begin{array}{l}1973: \text { FrP (5\%) } \\
1989: 13 \%\end{array}$ & \\
\hline $\mathbf{1 9 9 0 - 2 0 0 0}$ & & $\begin{array}{l}1995: \text { DF } \\
1998: 7,4 \%\end{array}$ & $\begin{array}{l}1993: 6,3 \% \\
1997: 15 \%\end{array}$ & $\begin{array}{l}1995: \text { PSM } \\
1999: 1 \%\end{array}$ \\
\hline $\mathbf{2 0 0 0 - 2 0 1 0}$ & $2006: 3 \%$ & $2001: 12 \%$ & $2005: 22 \%$ & $2007: 4 \%$ \\
& $2010: 5,7 \%$ & $2005: 14 \%$ & $2009: 23 \%$ & \\
& & $2007: 13,8 \%$ & & $2011: 19 \%$ \\
& & $2011: 12,3$ & $2013: 16,3 \%$ & $2015: 17,7 \%$ \\
\hline $\mathbf{2 0 1 0 - 2 0 1 6}$ & $2014: 13 \%$ & $2015: 21 \%$ & $2017: 15,2 \%$ & $2 \%$ \\
& & & &
\end{tabular}

Suecia: SD (Svenska Demokraterne: demócratas suecos); Dinamarca: DF (Dansk Folkeparti: partido del pueblo danés); Noruega: FrP (Fremskrittspartiet: partido del progreso); Finlandia: PSM (Perussuomalaiset; desde 2014 partido de los finlandeses)

En Noruega, el FrP, fundado en 1973 como partido antiimpuestos, liberal y conservador (crítico con el fuerte estado de inspiración socialdemócrata y defensor de la libertad individual), ha ido incorporando el componente nacionalista y antiinmigración (defensa de una visión de la cultura tradicional noruega). A las elecciones de 2013 fue en coalición con el partido conservador Høyre (Derecha), y tras ganar, entró por primera vez en el gobierno.

En Finlandia, el PSM se creó en 1995 como evolución del partido rural finlandés, y tras las elecciones del 2015 entró en un gobierno de coalición de centro-derecha, gestionando cuatro de los catorce ministerios, pero ha sufrido rupturas internas y su apoyo en sondeos ha caído mucho.

Es difícil predecir el margen de crecimiento de estos partidos antiinmigración. Según la Encuesta Europea de Valores del 2014, Suecia y Noruega y en menor medida Finlandia y Dinamarca tenían las opiniones más positivas hacia la inmigración, junto Alemania y los Países Bajos, mientras las opiniones más negativas se daban en los países de la Europa central y del este.

\section{Nota metodológica}

Las principales fuentes de datos utilizadas son de carácter secundario, complementadas con algunos datos y observaciones primarias, como documentos políticos y de gobierno, e informes de comisiones oficiales. Las fuentes secundarias más relevantes provienen de una selección de literatura empírica y política sobre el tema. Sobre las recientes políticas de inmigración e integración en los países nórdicos existe una relativamente amplia literatura empírica. ${ }^{19}$ Sobre la influencia de los partidos antiinmigración hay también una suficiente literatura

\footnotetext{
${ }^{19} \mathrm{BECH}$, Emily C., BOREVI, Karin y MOURITSEN, Per, "A 'civic turn' in Scandinavian family migration policies? Comparing Denmark, Norway and Sweden" en Comparative Migration Studies vol. 5, no 1, 2017, p. 7; BREIDAHL, Karen N. "Scandinavian exceptionalism? Civic integration and labour market activation for newly arrived immigrants"en Comparative Migration Studies, vol. 5, no 2, 2017; BROCHMANN, Grete y HAGELUND, Anniken (ed.), Immigration policy and the Scandinavian welfare state 1945-2010, Palgrave Macmillan, Basingstoke, 2012.
} 
empírica, que ha sido a su vez contrastada y complementada con diversos datos públicos. Sobre los medios de comunicación y los nuevos medios digitales se usan algunos estudios de caso, actualizados y complementados con algunos datos y observaciones originales. Finalmente, sobre la comunidad investigadora y experta, la relativamente poca literatura empírica existente se complementa con datos e información sobre la posición y la contribución de los autores clave en cada país, en base principalmente a sus publicaciones y su relación o no de asesoramiento con el gobierno.

\section{Dinamarca: discurso popular e integración cultural ${ }^{20}$}

Políticamente tiene una tradición de fuerte democracia popular y pluralismo civil, que legitima que la voluntad popular de la mayoría del parlamento pueda ser priorizada frente a las obligaciones legales internacionales. Ideológicamente ha integrado las tradiciones liberal y socialdemócrata en un tipo de liberalismo social donde el ciudadano desarrolla su individualidad en una cohesionada comunidad étnica.

Las políticas de integración han tenido un recorrido ambiguo. En los años setenta y ochenta la mayoría de los partidos políticos y sindicatos eran escépticos sobre la inmigración, y preferían no tener que desarrollar políticas de integración. Esa postura era clara en los partidos de centro-derecha y liberales. El partido socialdemócrata apostaba por una humanitaria política de refugio y un multiculturalismo "temporal" sin políticas de integración asumiendo que los refugiados volverían a sus países. Esta postura fue criticada por dirigentes locales en los municipios con una creciente presencia de inmigrantes y refugiados, principalmente al oeste de Copenhague (Vestegen). Finalmente, el partido socialdemócrata cedió a las demandas internas y externas. ${ }^{21}$ En 1998 el gobierno de centro-izquierda liderado por el partido socialdemócrata aprobó una ley de integración que establecía la realización obligatoria de cursos gratuitos de introducción al idioma, cultura y conocimiento general en los tres primeros años, para poder optar a la ayuda económica de recién llegada de cuatro años. Esta ayuda económica fue establecida en una cantidad inferior a la ayuda de asistencia social general, lo que fue criticado entre otros por la agencia de refugiados de la ONU, y fue equiparada posteriormente.

Entre 2001 y 2011 el nuevo gobierno de centro-derecha liberal con el apoyo parlamentario del partido antiinmigración DF llevó a cabo un intenso proceso de reformas y regulaciones. En 2001 se creó el ministerio para inmigración e integración. En 2002 introdujo dos medidas obligatorias: un programa-ayuda de primera acogida de tres años, y la ayuda económica de inicio de cuatro años, esta última condicionada a haber trabajado mínimamente o a una actitud activa en el mercado de trabajo. Para optar a la residencia permanente y la ciudadanía se establecieron requisitos culturales (aprobar exámenes en idioma y en conocimiento general) y económicos (no haber tenido ayudas sociales en los últimos años).

\footnotetext{
${ }^{20}$ Para un análisis específico de Dinamarca: HEDETOFT, UIf "More than kin and less than kind: the Danish politics of ethnic consensus and the pluricultural challenge" en CAMPBELL, John L., HALL, John A. y PEDERSEN, Ove K. (ed.), National identity and the varieties of capitalism: The Danish experience, McGill-Queen's Press-MQUP, Montreal, 2006, pp. 398-430.

${ }^{21}$ TESFAYE, Mattias, Velkommen Mustafa - om 50 års socialdemokratisk udlæningepolitik (Bienvenido Mustafa. Sobre los 50 años de política de extranjería de la socialdemocracia), København, Gyldendal, 2017.
} 
Las estadísticas y los informes oficiales han enfatizado el porcentaje de origen no-occidental de las personas extranjeras. En el 2015 la población de extranjeros y descendientes era el $12,3 \%$ (9,5\% nacidos en el extranjero y casi un $3 \%$ como descendientes, sin incluir a las personas solicitantes de asilo). De este $12,3 \%$ el $7 \%$ eran de países no-occidentales. De 1986 a 2016 el grupo no-occidental pasó de 60000 a 314500 y los descendientes de 14500 a 138 000. La inmigración occidental comenzó a crecer desde mediados del 2000, especialmente con la incorporación a la UE de los países del este. El discurso antiinmigración se ha reforzado con la crítica a algunos efectos de la libre circulación de trabajadores en la UE sobre beneficios sociales, como la ayuda económica por hijo-as (cheque infantil) aunque éstos no residan en el país.

Del 2011 al 2015 el nuevo gobierno de centro-izquierda suavizó algunas de las medidas: eliminó el programa de ayuda económica inicial condicionada, y equiparó las cantidades a los niveles generales para toda la ciudadanía. En las elecciones de junio del 2015 el partido antiinmigración DF fue el segundo partido en votos, y el nuevo gobierno del partido liberal en minoría dependía de su apoyo parlamentario. En los primeros meses de otoño del 2015 refugiados sirios entraban desde Alemania andando por las autopistas del sur del país, muchos de ellos con la intención de llegar a Suecia. DF lideró las demandas de control policial de las fronteras, que el gobierno aceptó. En agosto de 2016 el gobierno anunció cuarenta y cuatro medidas en la política de refugiados y de integración. En octubre del 2016 el partido liberal firmó una alianza con el partido conservador y el nuevo partido liberal. Las políticas de inmigración e integración iban a ser las mismas. ${ }^{22}$ Para la primavera del 2017 eran más de 50 medidas, con dos direcciones: hacia una mayor restricción y exigencia, y hacia mayores condiciones para optar a derechos de ciudadanía. Destaca así la fuerte condicionalidad económico-cultural.

El discurso crítico y el endurecimiento de las políticas contra la inmigración principalmente no-occidental han alcanzado un alto grado de aceptación en la sociedad. Dinamarca tiene actualmente una de las políticas de integración más restrictivas de la UE. ${ }^{23}$ El caso danés indica una temprana movilización de las ideas críticas con la inmigración. Según Wren, ${ }^{24}$ en los años ochenta y noventa se desarrolló un discurso de racismo cultural, enfocado en una postura antimusulmán y antirefugiados, impulsado por tres factores principales: los grupos antiinmigración de extrema derecha, los medios de comunicación, y una tradición de investigación académica determinada culturalmente. La crisis internacional en 2006 provocada por la publicación de un dibujo sobre Mahoma fue un ejemplo de esa postura crítica con el islam.

En el sistema de partidos políticos, los grupos en contra de la inmigración han concen-

22 "El gobierno llevará a cabo un política de extranjería dura, consecuente y realista. Debemos ayudar al mundo, al mismo tiempo que cuidamos de nuestro país. Debe haber un equilibrio entre el idealismo y el realismo. Los daneses somos un pueblo abierto, que queremos ayudar a aquellos que lo necesitan. Es una tradición de la que tenemos que estar orgullosos. Al mismo tiempo tenemos que ser realistas. Los problemas de guerras, conflictos y pobreza que asolan el mundo no se solucionan dando la bienvenida a todos los extranjeros" (Traducción propia de la introducción del capítulo correspondiente a la política de extranjería). Disponible en: https://www. regeringen.dk/nyheder/her-er-det-nye-regeringsgrundlag/, [Consultado el 20 de abril del 2017].

${ }^{23}$ Según varios índices convencionales usados por GOODMAN, Sara W. y WRIGHT, Matthew, Does mandatory integration matter?.., op.cit., y por SIMONSEN, Kristina B., Does citizenship always..., op.cit.

${ }^{24}$ WREN, Karen. "Cultural racism: something rotten in the state of Denmark?" en Social \& Cultural Geography vol. 2, no 2, 2001, pp. 141-162. 
trado su apuesta por el partido DF, el cual ha ido aumentando en poder e influencia política; en 2016 se creó un nuevo partido (Nye Borgelige) con una mayor crítica antiinmigración. El discurso crítico con la inmigración ha sido acogido por otros partidos. Además de los partidos de centro-derecha liberales, el partido socialdemócrata ha ido también aceptando la idea de la inmigración como una amenaza al estado del bienestar, claramente desde la campaña de 2015; la reciente publicación del portavoz-responsable en el partido busca el consenso interno a favor de unas políticas de inmigración-integración más "realistas". ${ }^{25}$

En los medios de comunicación ha aumentado el cisma entre los medios que se pueden llamar multiculturalistas moderados o integracionistas y los antiinmigracionistas populistas. Entre los primeros están el ente público de radio-televisión (Danmarks Radio) y algunos medios de prensa escrita del entorno de centro-izquierda. Entre los segundos destacan los periódicos populares más leídos del país (Ekstra Bladet y BT), que pertenecen a los dos grandes grupos de medios escritos, Berlignske Tidende y JP/Politiken, que a su vez gestionan otros grandes periódicos que mantienen posturas intermedias y moderadas. Los nuevos medios digitales, como Den Korte Avis o $24 n y t$, son muy utilizados por las personas y grupos antiinmigración y antiislam.

Entre los investigadores y expertos el debate está cada vez menos polarizado dentro en un amplio espacio intermedio de idealismo moderado y realismo que acepta las políticas de integración. Un relevante académico como Hedetoft ${ }^{26}$ argumentaba en el 2006 que no había más alternativa que una política de integración mínimamente exigente en los ámbitos laborales y culturales; ya que el estado-nación de bienestar danés se basaba en un consenso étnico, el cual requería cierto nivel de asimilación o acomodación de las minorías étnicas in-

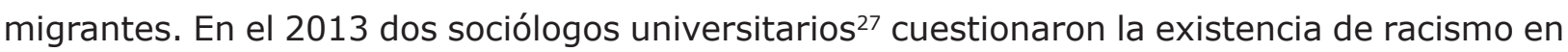
la sociedad danesa, desde una estrecha definición del fenómeno. Estos son dos ejemplos de argumentaciones e interpretaciones académicas justificativas de las políticas de integración.

En suma, Dinamarca presenta un discurso dominante crítico con la inmigración y exigente con la integración, cuyas políticas de refugio y asilo han recibido un número considerable de críticas de las Naciones Unidas. Las políticas han sido impulsadas por un fuerte partido político antiinmigración, difundidas por medios populistas y digitales, y legitimadas por un discurso académico prointegracionista. Su forma de integración cívica es altamente cultural con algunos elementos económicos y laborales.

\section{Suecia: discurso tecnocrático e integración política ${ }^{28}$}

Suecia es uno de los países europeos donde las políticas cívicas de integración han tenido un menor desarrollo. El país ha tenido históricamente una fuerte tradición humanitaria y multicultural, la cual era parte de la apuesta política internacional de la socialdemocracia sueca. La tecnocracia socialdemócrata construyó un marco discursivo fundamentado en una moderni-

\footnotetext{
25 TESFAYE, Mattias, Velkommen Mustafa..., op.cit.

${ }^{26}$ HEDETOFT, UIf, More than kin ..., op.cit.

$27 \mathrm{BECH}$, Henning y NECEF, Mehmet Ümit, Er danskerne racister?, Frydenlund, Copenhague, 2013.

${ }^{28}$ Para un análisis especifico de Suecia: GEDDES, Andrew y SCHOLTEN, Peter, "Sweden: immigration politics in an advanced welfare state" en GEDDES, Andrew y SCHOLTEN, Peter The politics of migration and immigration in Europe, Sage, London, 2016, pp. 125-145.
} 
dad liberal democrática donde los derechos humanos eran la referencia moral de la política. Esto generó una cultura de debate con un marcado peso de lo política y técnicamente correcto, que ha sido una de las causas de la baja politización del fenómeno, y que contrasta con una creciente opinión pública en la sociedad a favor de las políticas "cívicas" de integración.

Según Wickström, ${ }^{29}$ en los años sesenta y setenta Suecia pasó de una asimilación progresiva a un ideal multicultural de la mano de algunos líderes socialdemócratas como Olof Palme. Suecia había acogido a muchos trabajadores invitados y refugiados. En 1974 una comisión parlamentaria sobre inmigración estableció los tres principios de su modelo multicultural: igualdad respecto a los nativos suecos; libertad de elección que permitía el mantenimiento de la identidad cultural grupal; y colaboración y solidaridad entre nativos y recién llegados. Este fuerte ideal multicultural y humanitario quedó fuertemente enraizado en el debate político y tecnocrático, y ha limitado el desarrollo de la opinión pública contra la inmigración.

A finales de los ochenta se comenzó lentamente a alterar este modelo multicultural. En 1986 se eliminó el principio de protección de los derechos de las minorías culturales, y la población extranjera pasó a ser compuesta por individuos culturalmente diferentes; se argumentaba que una fuerte política multicultural reforzaba la marginalización de los grupos inmigrantes. Entre 1993 y 1997 se estableció la ayuda de introducción, a ser implementada por los municipios como una opción voluntaria y alternativa a la tradicional ayuda de asistencia social. En 1994 se introdujo el principio de autoestablecimiento residencial para los solicitantes de asilo, lo que estimuló la concentración de inmigrantes en áreas urbanas marginadas. En 1997 se limitó la opción de reagrupamiento familiar a la familia nuclear.

En 2006 un nuevo gobierno de centro-derecha liberal creó el ministerio de integración e igualdad de género. En 2007 se creó una comisión de análisis y de recomendaciones políticas. En el 2010 se aprobó ofrecer educación y ayuda a los inmigrantes de cara al mercado laboral. La política de empleo y laboral fue usada para solucionar los problemas socio-económicos y de integración de los inmigrantes. En el 2010 también se introdujo un requisito de autosuficiencia económica del solicitante para la reunificación familiar. Tras las elecciones del 2010 el gobierno de centro-derecha implementó los cambios que había propuesto en la legislatura anterior. El tema de la inmigración quedó integrado en el ministerio de justicia. En 2013 se produjeron muchos incidentes violentos protagonizadas por jóvenes mayormente descendientes de inmigrantes en zonas o barrios marginados, un fenómeno que se había dado en años anteriores en localidades como Södertälje, Malmö, Rosengård y Rinkeby. Según Fredlund-Blomst, ${ }^{30}$ las causas profundas de estas revueltas eran una combinación de condiciones económicas y laborales, y los efectos de las políticas de inmigración y las políticas de vivienda.

En 2015 y 2016, en el contexto de la llegada masiva de refugiados y de solicitudes de asilo, y el aumento del apoyo electoral al partido antiinmigración, el nuevo gobierno de

\footnotetext{
${ }^{29}$ WICKSTRÖM, Mats, "Conceptual change in postwar Sweden: the marginalization of assimilation and the introduction of integration" en HELLSTRÖM, Anders (ed). Debating multiculturalism in the Nordic welfare states, Palgrave Macmillan UK, 2013, Basingstoke, pp. 110-139.

${ }^{30}$ FREDLUND-BLOMST, Sofie, Assessing Immigrant Integration in Sweden after the May 2013 Riots, January 2014. Disponible en: http://www.migrationpolicy.org, [Consultado el 3 de marzo de 2017]
} 
centro-izquierda con el apoyo del resto de partidos de centro-derecha aprobó una serie de medidas.

Algunas fueron de urgencia y presentadas con un carácter temporal mientras el flujo de refugiados fuera alto. Otras tenían carácter de ley con un calendario más largo. En octubre del 2015 se aprobó que a los solicitantes de asilo solo se les concedería permisos temporales de residencia (tres años para los acogidos según la convención de Ginebra, y un año para los otros casos de necesaria protección); de esta decisión quedaban excluidos los refugiados de cuota, los menores no acompañados y las familias con niños. En noviembre se aprobaron nuevas medidas como el control de las fronteras y un ajuste de la política de asilo al mínimo que permitía la regulación de la UE; por ejemplo, el derecho al reagrupamiento familiar solo era concedido a los refugiados de la convención de Ginebra. Desde enero del 2016 se estableció control policial de las fronteras, y en la primavera y verano se aprobaron nuevas reglas, que afectaban principalmente a la reagrupación familiar (reducción del derecho al reagrupamiento familiar con un mayor número de condiciones) y a las condiciones de asilo (se establece solo el permiso temporal de residencia de 1 o 3 años).

En el debate sobre la inmigración hay una significativa distancia entre la opinión pública en la sociedad y la opinión político-tecnocrática. Dentro de un tono general de opinión humanitaria y multicultural, la sociedad ha indicado un creciente deseo de políticas de integración mientras la opinión político-tecnocrática se ha mantenido en posturas de moderado multiculturalismo formal, reflejado en uno de los más fáciles accesos a la ciudadanía ${ }^{31}$.

En el sistema de parditos políticos, SD ha tenido un creciente apoyo y presencia. Sus resultados en las elecciones de 2014 superaron los sondeos de opinión. El ser marginado por los otros partidos le permite presentarse como una auténtica oposición política al sistema establecido de partidos y tecnocracia. La llegada masiva de refugiados en 2015 llevó al gobierno de centro-izquierda bajo liderazgo social-demócrata a indicar que tenía el control de la situación y que debía detener el flujo de solicitudes de asilo y de refugiados; justificó estas decisiones con argumentos de temporalidad y de excepcionalidad.

En los medios de comunicación el debate se ha polarizado, y entre periodistas y expertos han aumentado las acusaciones de racismo, extremismo y radicalidad, por un lado, y de autocensura y de multiculturalismo elitista, por el otro. Los medios públicos son acusados por los grupos antiinmigración de sesgos favorables a las posturas de izquierda y multiculturales. Un grupo de periodistas (de medios de izquierda como el Dagens ETC) y de investigadores publicaba en el 2015 un informe sobre los mitos y las mentiras del discurso anti-inmigración. Al mismo tiempo, un creciente número de medios escritos con orientación de centro y centro-derecha han ido problematizando la cuestión de la inmigración. Por su parte, los medios digitales, como Facebook, Twitter, etc. han sido el canal de las mayores críticas a la inmigración y los refugiados. ${ }^{32}$ El grupo antiinmigración Soldiers of Odin puede ser el más preocu-

\footnotetext{
${ }^{31}$ Según el estudio de GOODMAN, Sara y WRIGHT, Mathew, Does mandatory integration..., op cit.

32 https://www.cnet.com/news/refugee-crisis-europe-social-media-impact-on-sweden-finland [consultado el 21 de agosto de 2017]
} 
pante, dado su carácter totalitario y fascista-vigilante. ${ }^{33}$

La mayoría de académicos y expertos siguen defendiendo cierto nivel de multiculturalismo y las políticas humanitarias. Jørgensen ${ }^{34}$ subraya cómo el dominante discurso académico, muy influyente en los informes y comisiones parlamentarias, ha usado los argumentos de discriminación estructural y sicológica por parte de la propia sociedad sueca. Otras voces critican el efecto negativo de este pensamiento-consenso tecnocrático y académico. En diciembre de 2016, un economista sueco de origen kurdo-iraní, Tino Sanandaji, economista investigador del sistema público y crítico con el discurso dominante, publicó el libro El gran reto. La política económica contra la exclusión y el comportamiento antisocial con datos cuantitativos sobre la conducta antisocial, criminalidad, etc. en "guetos", y sobre el coste de la política de inmigración y de refugiados; las altas ventas del libro indican la creciente aceptación de estas ideas.

En suma, Suecia presenta un discurso dominante de multiculturalismo decreciente y moderado, donde el gobierno ha ido adoptando políticas "cívicas" de integración y de mayor control de la inmigración de refugiados, presionado por un creciente partido antiinmigración que al parecer ha empezado a socavar el poder cultural o "definitorio" de la tecnocracia pública socialdemócrata y del mundo académico, promoviendo una idea de inevitabilidad de las nuevas políticas. Su modelo de integración es todavía altamente político, con uno de los accesos más generosos a la ciudadanía en la UE.

\section{Noruega: discurso político e integración social ${ }^{35}$}

Noruega combina elementos de Dinamarca y de Suecia. Respecto a la inmigración adoptó inicialmente el modelo de la política multicultural de Suecia, enmarcado en una filosofía cristiana de solidaridad humanitaria. De Dinamarca ha tomado recientemente inspiración para sus políticas de integración, como los requisitos laborales y culturales para acceder a la ciudadanía, pero en el último año Noruega ha inspirado también al gobierno danés, como se indica abajo.

Las políticas "cívicas" de integración comenzaron en los primeros años del siglo XXI. En el 2004, cuando el FrP tenía el apoyo del 20-25\% del electorado, se aprobó una ley de introducción que establecía un programa-ayuda para los recién llegados con una duración de dos años. La justificación era la importancia de la autosuficiencia económica como principio de una ciudadanía activa y de las nuevas políticas de bienestar. Posteriormente la participación en este programa-ayuda fue considerado una condición para optar a la residencia permanente y la ciudadanía. En el 2004 una comisión de expertos realizó propuestas que fueron aprobadas entre 2007 y 2010 por gobiernos de centro-izquierda liderados por el partido socialdemócrata.

33 TURTA, E., Frivilliga samhällshjältar i strid mot samhällskollaps-En kvalitativ innehållsanalys av diskussioner om organisationen Soldiers of Odin på Flashback och Facebook, 2016. Disponible en: http://hdl.handle. net/2077/48422 [consultado el 18 de agosto del 2017]

34 JØRGENSEN, Martin Bak, Understanding the research-policy nexus..., op.cit.

${ }^{35}$ Para un análisis específico de Noruega: BROCHMANN, Grete y HAGELUND, Anniken "Norway: The Land of the Golden Mean" en BROCHMANN, Grete y HAGELUND, Anniken (ed.), Immigration policy and the Scandinavian welfare state 1945-2010, Palgrave Macmillan, Basingstoke, 2012, pp. 149-223. 
Los atentados del extremista Brevik en julio de 2011, en donde murieron setenta y siete personas, generaron un fuerte debate. Brevik, antiguo afiliado al FrP, atentó contra la juventud del partido socialdemócrata acusándola de estar promoviendo la multiculturalidad. Tras un periodo de autocontrol las posturas críticas contra la inmigración continuaron extendiéndose. En las elecciones del 2013 el FrP, aunque descendió en apoyo electoral al 16\% con 29 escaños, entró en el gobierno de coalición con el partido derecha (48 escaños), con apoyo parlamentario del partido cristiano y del liberal, con 10 y 9 escaños. En diciembre de 2015, una de las líderes del ala radical y conservadora del FrP, Listhaug, se hizo cargo del nuevo ministerio de migración e integración. En el contexto del creciente número de solicitudes de asilo, en 2016 el gobierno adoptó medidas que incluían nuevas condiciones para acceder a derechos sociales y políticos. Las restricciones y requisitos para la reagrupación familiar también aumentaron, tanto para solicitantes como para familiares. El gobierno estableció acuerdos bilaterales con algunos países para la deportación de solicitantes de asilo, algo que el gobierno danés ha empezado a imitar.

En el sistema de partidos políticos, el FrP, desde 2013 en el gobierno de coalición, y desde diciembre del 2015 dirigiendo el ministerio de inmigración. La ministra, Listaug, había criticado la ingenua moral cristiana como una especie de "tiranía de la bondad". Su discurso de integración es de asimilacionismo, y pide que los inmigrantes adopten los valores y las prácticas culturales noruegas. La reciente crisis de refugiados le permitió justificar un discurso más duro y "realista", aunque no pudo aprobar en el parlamento todas sus propuestas. En las elecciones de septiembre del 2017 obtuvo el 15,2\% de los votos y se mantiene en la coalición de gobierno.

En los medios de comunicación ha aumentado el número de actores y opiniones, a los dos lados del discurso dominante liderado por los medios públicos con posturas de multiculturalismo moderado. Por un lado, según Wiggen, ${ }^{36}$ el discurso antiinmigración fue ganando espacio y medios y académicos cercanos al FrP promovieron miedos, mitos y críticas sobre los inmigrantes. Por otro lado, en el 2011 la opinión mayoritaria de la elite pública, que todavía silenciaba el discurso del FrP, empezaba a recibir una creciente respuesta de personas de minorías étnicas y de voces en defensa de la población inmigrante. ${ }^{37}$

El discurso académico tiene un papel clave. Litman ${ }^{38}$ subraya el papel de algunos intelectuales y académicos de izquierda en la defensa de la necesidad de reducir la inmigración y los aspectos negativos del multiculturalismo, dentro de su colaboración experta con el gobierno. En los acuerdos parlamentarios de diciembre de 2015 se aprobó el encargo de un estudio con el objetivo de mejorar la integración y de contener la entrada masiva de inmigrantes y refugiados. En el informe, hecho público a comienzos de $2017,{ }^{39}$ se revisa la

36 WIGGEN, Mette. "Rethinking anti-immigration rhetoric after the Oslo and Utøya terror attacks" en New Political Science vol. 34, no 4, 2012, pp. 585-604.

${ }^{37}$ FIGENSCHOU, Tine Ustad y BEYER, Audun. "Elitene, minoritetene og mediene-Definisjonsmakt i norsk innvandringsdebatt" en Tidsskrift for Samfunnsforskning, vol. 55, no 1, pp. 23-51, 2014.

38 LITHMAN, Yngve. "Norwegian multicultural debates in a Scandinavian comparative perspective" en HELLSTRÖM, Anders (ed.), Debating multiculturalism in the Nordic welfare states, Palgrave Macmillan UK, Basingstoke, 2013, pp. 246-269.

${ }^{39} \mathrm{NOU}$ (2017) "Integrasjon og tillit. Langsiktige konsekvenser av høy innvandring (Integración y confianza. Consecuencias a largo plazo de la alta inmigración)", Gobierno de Noruega. 
literatura académica y se concluye que hay un acuerdo respecto a la presión de la inmigración sobre el estado de bienestar nórdico, y un cuestionamiento de la capacidad del mismo para integrar a un alto número de inmigrantes. En el capítulo $10^{40}$ se afirma el doble objetivo de las políticas de integración: mejorar la integración y hacer Noruega menos atractiva para la inmigración. En el capítulo 11 se presentan los tres escenarios o estrategias de integración en una situación de llegada masiva de inmigrantes y refugiados: de universalismo basado en derechos, de ajuste a través de los mercados, o de inversión social. Los tres escenarios se alejan del anterior modelo multicultural.

En suma, Noruega presenta un discurso dominante políticamente moderado, pero crecientemente prointegracionista y crítico con la inmigración, enfatizando los límites de su estado de bienestar para integrar a inmigrantes. Estas ideas han sido impulsadas por un partido antiinmigración en el gobierno desde el 2013, y por la función legitimadora de un colectivo investigador acostumbrado a colaborar con los gobiernos. Su modelo de integración es de carácter más social, al enfatizar las condiciones socio-laborales para acceder a la ciudadanía.

\section{Finlandia: un caso emergente}

Finlandia fue una colonia de Rusia y Suecia que obtuvo su independencia en 1905. De fuerte tradición emigratoria, ha comenzado a recibir inmigración: en el 2013 la población extranjera era el $5,5 \%$, y el $2 \%$ eran de países miembros de la UE, y un 1,5\% era de la antigua Unión Soviética. Tradicionalmente han existido dos minorías culturales que han recibido un tratamiento especial: los lapones en el norte y la comunidad sueca. El partido antiinmigración se ha centrado en la cuestión de los derechos de las minorías culturales. ${ }^{41}$ Las políticas de integración son nuevas, pero el discurso antiinmigración está emergiendo rápidamente. Horsti y Nikunen ${ }^{42}$ enfatizan el doble efecto de los medios digitales de comunicación: influir y moldear la agenda de los medios de comunicación tradicionales, y la popularización nacionalista del debate sobre la inmigración. Mäkinen ${ }^{43}$ destaca el papel de la plataforma digital Hommaforum, donde el activismo antiinmigración combina el anonimato y la participación de líderes políticos para promover una segregación de los inmigrantes como los gitanos de la Europa central y del este.

\section{Conclusiones}

Para comprender las recientes políticas de inmigración-integración en los países nórdicos se han observado principalmente las dinámicas y discursos políticos dominantes que han impulsado y promovido las nuevas políticas "cívicas" de integración, dentro de las concepciones nacionales de ciudadanía e integración. Se han analizado tres actores principales en la construc-

40 "La comisión ha centrado su análisis en las políticas de ayudas sociales, vivienda e integración, y ha analizado elementos relevantes de las políticas educativas, laborales y de bienestar; estos aspectos son considerados clave para la capacidad de integración. Un aspecto relevante a evaluar es cómo se pueden reducir las barreras al trabajo o al estudio para los refugiados, sin que eso suponga (con cierta probabilidad) hacer Noruega más atractiva para los solicitantes de asilo" (Traducción propia)

${ }^{41}$ WAHLBECK, Öste, "True Finns and Non-True Finns: The Minority Rights Discourse of Populist Politics in Finland" en Journal of Intercultural Studies, vol. 37, no 6, 2016, pp. 574-588.

42 HORSTI, Karina y NIKUNEN, Kaarina, "The ethics of hospitality in changing journalism: A response to the rise of the anti-immigrant movement in Finnish media publicity" en European journal of cultural studies vol. 16, no 4, 2013, pp. 489-504.

43 MÄKINEN, Katariina. "Struggles of citizenship and class: anti-immigration activism in Finland" en The Sociological Review, 2016, pp. 1-17. 
ción de esos discursos y políticas: los partidos antiinmigración, los medios de información y especialmente los medios populistas, sociales y digitales, y la comunidad académica-experta.

Cada país nórdico tiene su particularidad en las políticas de inmigración e integración. Dinamarca comenzó tempranamente a endurecer sus políticas, llegando a uno de los mayores niveles de restricción en la UE. Suecia ha mantenido, con crecientes dificultades, su tradición humanitaria y de multiculturalidad. Noruega ha pasado recientemente de un modelo multicultural de inspiración sueco a adoptar el modelo danés con políticas cada vez más duras. Entre estos países ha habido un alto grado de observación e influencia mutua en sus políticas.

Los países nórdicos representan un caso de inmigración-integración en estados-nación avanzados, culturalmente homogéneos y socialmente cohesionados, donde la inmigración-integración es percibida de forma problemática respecto al sistema público de bienestar. Sus solidarias políticas internacionales (ayuda al desarrollo, política de refugiados, etc.) han traído a estos países una creciente diversidad cultural. Esta situación, en la actual era neoliberal y neonacionalista, ha provocado conflictos ideológicos y políticos sobre la inmigración, cuya solución pasa a nivel nacional por cuidadosos discursos y mejores gestiones político-administrativas, y por una mejor coordinación transnacional e internacional.

En estos países la gestión de la crisis de refugiados de 2014-15 trajo un mayor control de las fronteras y de entradas de personas (limitando el espacio Schengen), y un endurecimiento en las solicitudes y las condiciones de los refugiados. Algunas de las nuevas medidas adoptadas han venido probablemente para quedarse, dentro de la falta de coordinación en la UE para gestionar estos movimientos forzados de población. La inmigración forzada y económica-voluntaria no parece que vaya a descender, y los países europeos, receptores de la misma, han aumentado su fortificación mediante estas nuevas políticas de inmigración e integración.

\section{Bibliografía}

ANDERSSON, Mette, "The debate about multicultural Norway before and after 22 July 2011" en Identities: Global Studies in Culture and Power 289 vol. 19, no 4, 2012, pp. 418- 427.

$\mathrm{BECH}$, Henning y NECEF, Mehmet Ümit, Er danskerne racister?, Frydenlund, Copenhague, 2013.

BECH, Emily C., BOREVI, Karin y MOURITSEN, Per, "A 'civic turn' in Scandinavian family migration policies? Comparing Denmark, Norway and Sweden" en Comparative Migration Studies vol. 5, no 1, 2017, p. 7.

BERGMANN, Eirikur. Nordic Nationalism and Right-Wing Populist Politics, Palgrave Macmillan UK, Londres, 2017.

BREIDAHL, Karen N., "Scandinavian exceptionalism? Civic integration and labour market activation for newly arrived immigrants" en Comparative Migration Studies, vol. 5, nº 2, 2017.

BROCHMANN, Grete y HAGELUND, Anniken "Norway: The Land of the Golden Mean" en BROCHMANN, Grete y HAGELUND, Anniken (ed.). Immigration policy and the Scandinavian welfare state 19452010, Palgrave Macmillan, Basingstoke, 2012, pp. 149-223.

BROCHMANN, Grete y HAGELUND, Anniken, "Migrants in the Scandinavian welfare state. The emergence of a social policy problem" en Nordic Journal of Migration Research, vol. 1, no 1, 2011, pp. 13-24.

CASTLES, Stephen, "Towards a sociology of forced migration and social transformation" en Sociology, vol. 37, no 1,2003 , pp. 13-34.

ELLINAS, Antonis, The Media and the Far Right in Western Europe: Playing the Nationalist Card, Cambridge University Press, Cambridge, 2010.

EMILSON, Henrik, "A national turn of local integration policy: multi-level governance dynamics in Denmark and Sweden" en Comparative Migration Studies vol. 3.1, n 7, 2015. 
FIDDIAN-QASMIYEH, Elena, LOESCHER, Gil, LONG, Katy y SIGONA, Nando (ed.) The Oxford handbook of refugee and forced migration studies, Oxford University Press, Oxford, 2014

FIGENSCHOU, Tine Ustad y BEYER, Audun. "Elitene, minoritetene og mediene-Definisjonsmakt i norsk innvandringsdebatt", Tidsskrift for Samfunnsforskning, vol. 55, no 1, 2014, pp. 23-51.

FREUDLUN-BLOMST, Sofie, Assessing Immigrant Integration in Sweden after the May 2013 Riots, 2014, January: http://www.migrationpolicy.org [Consultado el 3 de marzo de 2017)

GARDELL, Mattias. "What's Love Got to Do with It?: Ultranationalism, Islamophobia, and Hate Crime in Sweden" en Journal of Religion and Violence, 2015.

GEDDES, Andrew y SCHOLTEN, Peter, The politics of migration and immigration in Europe. Sage, London, 2016.

GEDDES, Andrew y SCHOLTEN, Peter, "Sweden: inmigration politics in an advanced welfare state", en GEDDES, Andrew y SCHOLTEN, Peter, The politics of migration and immigration in Europe. Sage, London, 2016, pp. 125-145.

GOODMAN, Sara W. "Fortifying citizenship: policy strategies for civic integration in Western Europe" en World Politics vol. 64, no 04, 2012, pp. 659-698.

GOODMAN, Sara W. y WRIGHT, Matthew "Does mandatory integration matter? Effects of civic requirements on immigrant socio-economic and political outcomes" en Journal of Ethnic and Migration Studies vol. 41 , no 12, 2015, pp. 1885-1908.

HEDETOFT, UIf Riber, "More than kin and less than kind: the Danish politics of ethnic consensus and the pluricultural challenge" en CAMPBELL, John L., HALL, John A. y PEDERSEN, Ove K. (ed.) National identity and the varieties of capitalism: The Danish experience, McGill-Queen's Press-MQUP, 2006, pp. 398-430.

HELSSTRÖM, Anders, Trust us: Reproducing the nation and the Scandinavian nationalist populist parties, Berghahn Books, 2016.

HORSTI, Karina y NIKUNEN, Kaarina, "The ethics of hospitality in changing journalism: A response to the rise of the anti-immigrant movement in Finnish media publicity" en European journal of cultural studies vol. 16, no 4, 2013, pp. 489-504.

KING, Russell, Theories and typologies of migration: An overview and a primer. Malmö University, Malmö Institute for Studies of Immigration, Diversity and Welfare (MIM), 2013.

JOPPKE, Christian, "Beyond national models: Civic integration policies for immigrants in Western Europe" en West European Politics vol. 30, no 1, 2007, pp. 1-22.

JØRGENSEN, Martin Bak, "Understanding the research-policy nexus in Denmark and Sweden: The field of migration and integration" en The British Journal of Politics and International Relations vol. 13, no 1, 2011, pp. 93-109.

LITHMAN, Yngve, "Norwegian multicultural debates in a Scandinavian comparative perspective" en HELLSTRÖM, Anders (ed), Debating multiculturalism in the Nordic welfare states, Palgrave Macmillan UK, Basingstoke, 2013, pp. 246-269.

MÄKINEN, Katariina, "Struggles of citizenship and class: anti-immigration activism in Finland" en The Sociological Review, 2016, pp. 1-17.

MESSINA, Anthony M., The logics and politics of post-WWII migration to Western Europe, Cambridge University Press, Cambridge, 2007.

NOU (2017) "Integrasjon og tillit. Langsiktige konsekvenser av høy innvandring (Integración y confianza. Consecuencias a largo plazo de la alta inmigración)", Gobierno de Noruega.

SCHOLTEN, Peter, ENTZINGER, Han, PENNINX, Rinus, "Research-policy dialogues on migrant integration in Europe: comparison and conclusions", en SCHOLTEN, Peter y ENTZINGER, Han (ed) Integrating Immigrants in Europe. Springer International Publishing, 2015, pp. 315-336.

SIMONSEN, Kristina Bakkær, "Does citizenship always further immigrants' feeling of belonging to the host nation? A study of policies and public attitudes in 14 Western democracies" en Comparative Migration Studies vol. 5.1, no 3, 2016.

VAN DIJK, Teun, "Ideologies, racism, discourse: Debates on immigration and ethnic issues" en WAL Jessika ter y VERKUYTEN, Maykel (ed.), Comparative perspectives on racism, Ashgate, 2000, pp. 91-116.

VAN DIJK, Teun, "Discurso de las élites y racismo institucional" en LARIO, Manuel (ed.). Medios de comunicación e inmigración, CAM - Obra Social, Murcia, 2006, pp. 15-34.

WAHLBECK, Öste, "True Finns and Non-True Finns: The Minority Rights Discourse of Populist Politics in Finland" en Journal of Intercultural Studies, vol. 37, n6, 2016, pp. 574-588.

WICKSTRÖM, Mats, "Conceptual change in postwar Sweden: the marginalization of assimilation and the introduction of integration" en HELLSTRÖM, Anders (ed.). Debating multiculturalism in the Nordic welfare states, Palgrave Macmillan UK, 2013, 110-139.

WIGGEN, Mett, "Rethinking anti-immigration rhetoric after the Oslo and Utøya terror attacks" en New Political Science vol. 34, no 4, 2012, pp. 585-604.

WREN, Karen, "Cultural racism: something rotten in the state of Denmark?" en Social \& Cultural Geography, vol. 2, no 2, 2001, pp. 141-162. 


\section{RELACIONES INTERNACIONALES}

Revista académica cuatrimestral de publicación electrónica Grupo de Estudios de Relaciones Internacionales (GERI) Universidad Autónoma de Madrid, España

www.relacionesinternacionales.info

ISSN 1699 - 3950

ff facebook.com/RelacionesInternacionales

twitter.com/RRInternacional 